\title{
Continuous Maps on Digital Simple Closed Curves
}

\author{
Laurence Boxer ${ }^{1,2}$ \\ ${ }^{1}$ Department of Computer and Information Sciences, Niagara University, New York, USA \\ ${ }^{2}$ Department of Computer Science and Engineering, State University of New York at Buffalo, Buffalo, USA \\ E-mail:boxer@niagara.edu \\ Received August 2, 2010; revised September 14, 2010; accepted September 16, 2010
}

\begin{abstract}
We give digital analogues of classical theorems of topology for continuous functions defined on spheres, for digital simple closed curves. In particular, we show the following: 1) A digital simple closed curve $S$ of more than 4 points is not contractible, i.e., its identity map is not nullhomotopic in $S$;2) Let $X$ and $Y$ be digital simple closed curves, each symmetric with respect to the origin, such that $|Y|>5$ (where $|Y|$ is the number of points in $Y$ ). Let $f: X \rightarrow Y$ be a digitally continuous antipodal map. Then $f$ is not nullhomotopic in $Y$; 3) Let $S$ be a digital simple closed curve that is symmetric with respect to the origin. Let $f: S \rightarrow Z$ be a digitally continuous map. Then there is a pair of antipodes $\{x,-x\} \subset S$ such that $|f(x)-f(-x)| \leq 1$.
\end{abstract}

Keywords: Digital Image, Digital Topology, Homotopy, Antipodal Point

\section{Introduction}

A digital image is a set $X$ of lattice points that model a "continuous object" $Y$, where $Y$ is a subset of a Euclidean space. Digital topology is concerned with developing a mathematical theory of such discrete objects so that, as much as possible, digital images have topological properties that mirror those of the Euclidean objects they model; however, in digital topology we view a digital image as a graph, rather than, e.g., a metric space, as the latter would, for a finite digital image, result in a discrete topological space. Therefore, the reader is reminded that in digital topology, our "nearness" notion is the graphical notion of adjacency, rather than a neighborhood system as in classical topology; usually, we use one of the natural $c_{l}$-adjacencies (see Section 2). Early papers in the field, e.g., [1-8], noted that this notion of nearness allows us to express notions borrowed from classical topology, e.g., connectedness, continuous function, homotopy, and fundamental group, such that these often mirror their analogs with respect to Euclidean objects modeled by respective digital images. Applications of digital topology have been found shape description and in image processing operations such as thinning and skeletonization [9].

A. Rosenfeld wrote the following: "The discrete grid (of pixels or voxels) used in digital topology can be regarded as a 'digitization' of (two or three-dimensional)
Euclidean space; from this viewpoint, it is of interest to study conditions under which this digitization process preserves topological (or other geometric) properties" [3].

In this spirit, we obtain in this paper several properties of continuous maps on digital simple closed curves, inspired by analogs for Euclidean simple closed curves. In particular, we show that digital simple closed curves of more than 4 points are not contractible, and we obtain several results for continuous maps and antipodal points on digital simple closed curves.

\section{Preliminaries}

Let $Z$ be the set of integers. Then $Z^{d}$ is the set of lattice points in $d$-dimensional Euclidean space. Let $X \subset Z^{d}$ and let $\kappa$ be some adjacency relation for the members of $X$. Then the pair $(X, \kappa)$ is said to be a (binary) digital image. A variety of adjacency relations are used in the study of digital images. Well known adjacencies include the following.

For a positive integer $l$ with $1 \leq l \leq d$ and two distinct points $p=\left(p_{1}, p_{2}, \ldots, p_{d}\right), q=\left(q_{1}, q_{2}, \ldots, q_{d}\right) \in Z^{d}, \quad p$ and $q$ are $c_{l}$-adjacent [10] if

- there are at most $l$ indices $i$ such that $\left|p_{i}-q_{i}\right|=1$, and

- for all other indices $j$ such that $\left|p_{j}-q_{j}\right| \neq 1$, $p_{j}=q_{j}$. See Figure 1. 


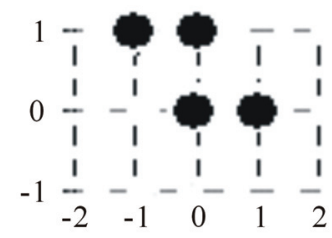

Figure 1. In $Z^{2}$, each of the points $p_{1}=(1,0)$ and $p_{2}=(0,1)$ is both $c_{1}$-adjacent and $c_{2}$-adjacent to $p_{0}=(0,0)$, since both $p_{1}$ and $p_{2}$ differ from $\boldsymbol{p}_{0}$ by 1 in exactly one coordinate and coincide in the other coordinate; $p_{3}=(-1,1)$ is $c_{2}$-adjacent, but not $c_{1}$-adjacent, to $p_{0}$, since $p_{0}$ and $p_{3}$ differ by 1 in both coordinates.

The notation $c_{l}$ is sometimes also understood as the number of points $q \in Z^{d}$ that are $c_{l}$-adjacent to a given point $p \in Z^{d}$. Thus, in $Z$ we have $c_{1}=2$; in $Z^{2}$ we have $c_{1}=4$ and $c_{2}=8$; in $Z^{3}$ we have $c_{1}=6, c_{2}=18$, and $c_{3}=26$.

More general adjacency relations are studied in [11]. Let $\kappa$ be an adjacency relation defined on $Z^{d}$. A $\kappa$ neighbor of a lattice point $p$ is $\kappa$-adjacent to $p$. A digital image $X \subset Z^{d}$ is $\kappa$-connected [11] if and only if for every pair of different points $x, y \in X$, there is a set $\left\{x_{0}, x_{1}, \ldots, x_{r}\right\}$ of points of a digital image $X$ such that $x=x_{0}, y=x_{r}$ and $x_{i}$ and $x_{i+1}$ are $\kappa$-neighbors where $i \in\{0,1, \ldots, r-1\}$.

Let $a, b \in Z$ with $a<b$. A digital interval [7] is a set of the form

$$
[a, b]_{Z}=\{z \in Z \mid a \leq z \leq b\} .
$$

Let $X \subset Z^{d_{0}}$ and $Y \subset Z^{d_{1}}$ be digital images with $\kappa_{0}$-adjacency and $\kappa_{1}$-adjacency respectively. A function $f: X \rightarrow Y$ is said to be $\left(\kappa_{0}, \kappa_{1}\right)$-continuous $[2,8]$, if for every $\kappa_{0}$-connected subset $U$ of $X$, $f(U)$ is a $\kappa_{1}$-connected subset of $Y$. We say that such a function is digitally continuous.

Proposition 2.1 $[2,8]$ Let $X \subset Z^{d_{0}}$ and $Y \subset Z^{d_{1}}$ be digital images with $\kappa_{0}$-adjacency and $\kappa_{1}$-adjacency respectively. Then the function $f: X \rightarrow Y$ is $\left(\kappa_{0}, \kappa_{1}\right)$ continuous if and only if for every $\kappa_{0}$-adjacent points $\left\{x_{0}, x_{1}\right\}$ of $X$, either $f\left(x_{0}\right)=f\left(x_{1}\right)$ or $f\left(x_{0}\right)$ and $f\left(x_{1}\right)$ are $\kappa_{1}$-adjacent in $Y$.

This characterization of continuity is what is called an immersion, gradually varied operator, or gradually varied mapping in $[12,13]$.

Given digital images $\left(X_{i}, \kappa_{i}\right), i \in\{0,1\}$, suppose there is a $\left(\kappa_{0}, \kappa_{1}\right)$-continuous bijection $f: X_{0} \rightarrow X_{1}$ such that $f^{-1}: X_{1} \rightarrow X_{0}$ is $\left(\kappa_{1}, \kappa_{0}\right)$-continuous. We say $X_{0}$ and $X_{1}$ are $\left(\kappa_{0}, \kappa_{1}\right)$-isomorphic [14] (this was called $\left(\kappa_{0}, \kappa_{1}\right)$-homeomorphic in [7]) and $f$ is a $\left(\kappa_{0}, \kappa_{1}\right)$-isomorphism (respectively, a $\left(\kappa_{0}, \kappa_{1}\right)$-homeo- morphism).

By a digital $\kappa$-path from $x$ to $y$ in a digital image $X$, we mean a $(2, \kappa)$-continuous function $f:[0, m]_{Z} \rightarrow X$ such that $f(0)=x$ and $f(m)=y$. We say $m$ is the length of this path. A simple closed $\kappa$-curve of $m \geq 4$ points (for some adjacencies, the minimal value of $m$ may be greater than 4; see below) in a digital image $X$ is a sequence $\{f(0), f(1), \ldots$, $f(m-1)\}$ of images of the $\kappa$-path $f:[0, m-1]_{Z}$ $\rightarrow X$ such that $f(i)$ and $f(j)$ are $\kappa$-adjacent if and only if $j=(i \pm 1) \bmod m$. If $S=\left\{x_{i}\right\}_{i=0}^{m-1}$ where $x_{i}=f(i)$ for all $i \in[0, m-1]_{Z}$, we say the points of $S$ are circularly ordered.

Digital simple closed curves are often examples of digital images $X \subset Z^{d}$ for which it is desirable to consider $Z^{d} \backslash X$ as a digital image with some adjacency (not necessarily the same adjacency as used by $X$ ). For example, by analogy with Euclidean topology, it is desirable that a digital simple closed curve $X \subset Z^{2}$ satisfy the "Jordan curve property" of separating $Z^{2}$ into two connected components (one "inside" and the other "outside" $X)$. An example that fails to satisfy this property [10] if we allow $|X|=4$ is given by $\left(X, c_{1}\right)$, where

$$
X=\{(0,0),(1,0),(1,1),(0,1)\}
$$

is circularly ordered, and $\left(Z^{2} \backslash X, c_{2}\right)$ is $c_{2}$-connected. However, this anomaly is essentially due to the "smallness" of $X$ as a simple closed curve; it is known $[1,15,16]$ that for $m_{1}=8$ and $m_{2}=4$, if $X \subset Z^{2}$ is a digital simple closed $c_{i}$-curve such that $|X| \geq m_{i}$, then $Z^{2} \backslash X$ has exactly $2 \quad c_{3-i}$-connected components, $i \in\{1,2\}$. Thus, it is customary to require that a digital simple closed curve $X \subset Z^{2}$ satisfy $|X| \geq 8$ when $c_{1}$ adjacency is used; $|X| \geq 4$ when $c_{2}$-adjacency is used.

Let $X \subset Z^{d_{0}}$ and $Y \subset Z^{d_{1}}$ be digital images with $\kappa_{0}$-adjacency and $\kappa_{1}$-adjacency respectively. Two $\left(\kappa_{0}, \kappa_{1}\right)$-continuous functions $f, g: X \rightarrow Y$ are said to be digitally $\left(\kappa_{0}, \kappa_{1}\right)$-homotopic in $Y$ [8] if there is a positive integer $m$ and a function $H: X \times[0, m]_{Z} \rightarrow Y$ such that

- for all $x \in X, H(x, 0)=f(x)$ and $H(x, m)=g(x)$;

- for all $x \in X$, the induced function $H_{x}:[0, m]_{Z}$ $\rightarrow Y$ defined by

$H_{x}(t)=H(x, t)$ for all $t \in[0, m]_{Z}$,

is $\left(2, \kappa_{1}\right)$-continuous; and

- for all $t \in[0, m]_{Z}$, the induced function $H_{t}: X \rightarrow Y$ defined by

$H_{t}(x)=H(x, t)$ for all $x \in X$,

is $\left(\kappa_{0}, \kappa_{1}\right)$-continuous.

We say that the function $H$ is a digital $\left(\kappa_{0}, \kappa_{1}\right)-$ homotopy between $f$ and $g$. 
Additional terminology associated with homotopic maps includes the following.

- If $g$ is a constant map, we say $f$ is $\left(\kappa_{0}, \kappa_{1}\right)$ nullhomotopic [8]; if, further, $\left(X, \kappa_{0}\right)=\left(Y, \kappa_{1}\right)$ and $f=1_{X}$ (the identity map on $X$ ), we say $X$ is $\kappa_{0}$ contractible $[7,17]$.

- If $H\left(x_{0}, t\right)=f\left(x_{0}\right)$ for some $x_{0} \in X$ and all $t \in[0, m]_{Z}$, we say $H$ is a $\left(\kappa_{0}, \kappa_{1}\right)$-pointed homotopy [18].

- If $H:\left[0, m_{0}\right]_{Z} \times\left[0, m_{1}\right]_{Z} \rightarrow Y$ is a $\left(c_{1}, \kappa\right)$-homotopy between $\left(c_{1}, \kappa\right)$-continuous functions

$f, g:\left[0, m_{0}\right]_{Z} \rightarrow Y$ such that for all $t \in\left[0, m_{1}\right]_{Z}$, $H(0, t)=f(0)=g(0)$ and $H\left(m_{0}, t\right)=f\left(m_{0}\right)=g\left(m_{0}\right)$, we say $H$ holds the endpoints fixed [18].

Proposition 2.2 [8] Suppose $f_{0}, f_{1}: X \rightarrow Y$ are $(\kappa, \lambda)$-continuous and $(\kappa, \lambda)$-homotopic. Suppose $g_{0}, g_{1}: Y \rightarrow Z$ are $(\lambda, \mu)$-continuous and $(\lambda, \mu)$-homotopic. Then $g_{0} \circ f_{0}$ and $g_{1} \circ f_{1}$ are $(\kappa, \mu)$-homotopic in $Z$.

\section{Homotopy Properties of Digital Simple Closed Curves}

A classical theorem of Euclidean topology, due to L.E.J. Brouwer, states that a $d$-dimensional sphere $S^{d}$ is not contractible [19]. Theorem 3.3, below, is a digital analog, for $d=1$. We also present some related results in this section.

Proposition 3.1 Let $S_{a}$ be a digital simple closed $\kappa_{a}$-curve, $a \in\{0,1\}$. Let $f: S_{0} \rightarrow S_{1}$ be a $\left(\kappa_{0}, \kappa_{1}\right)$ continuous function. If $\left|S_{0}\right|=\left|S_{1}\right|$, then the following are equivalent.

a) $f$ is one-to-one.

b) $f$ is onto.

c) $f$ is a $\left(\kappa_{0}, \kappa_{1}\right)$-isomorphism.

Proof: Since $\left|S_{0}\right|=\left|S_{1}\right|$, the equivalence of a) and b) follows from the fact that $S_{0}$ is a finite set. That c) implies both a) and b) follows from the definition of isomorphism. Therefore, we can complete the proof by showing that b) implies c).

Let $S_{a}=\left\{x_{a, i}\right\}_{i=0}^{n-1}$, where the points of $S_{a}$ are circularly ordered, $a \in\{0,1\}$. Let $x_{1, u} \in S_{1}$ and let $x_{0, v}=f^{-1}\left(x_{1, u}\right)$. Then the $\kappa_{1}$-neighbors of $x_{1, u}$ in $S_{1}$ are $x_{1,(u-1) \bmod n}$ and $x_{1,(u+1) \bmod n}$, and the $\kappa_{0}$-neighbors of $x_{0, v}$ in $S_{0}$ are $x_{0,(v-1) \bmod n}$ and $x_{0,(v+1) \bmod n}$. Since $f$ is a continuous bijection, our choice of $x_{0, v}$ implies

$f\left(\left\{x_{0,(v-1) \bmod n}, x_{0,(v+1) \bmod n}\right\}\right)=\left\{x_{1,(u-1) \bmod n}, x_{1,(u+1) \bmod n}\right\}$.

Thus,

$f^{-1}\left(\left\{x_{1,(u-1) \bmod n}, x_{1,(u+1) \bmod n}\right\}\right)=\left\{x_{0,(v-1) \bmod n}, x_{0,(v+1) \bmod n}\right\}$.

Since $u$ was taken as an arbitrary index, $f^{-1}$ is $\left(\kappa_{1}, \kappa_{0}\right)$-continuous, so $f$ is a $\left(\kappa_{0}, \kappa_{1}\right)$-isomorphism.
Theorem 3.2 Let $S$ be a simple closed $\kappa$-curve and let $H: S \times[0, m]_{Z} \rightarrow S$ be a $(\kappa, \kappa)$-homotopy between an isomorphism $H_{0}$ and $H_{m}=f$, where $f(S) \neq S$. Then $|S|=4$.

Proof: Let $S=\left\{x_{i}\right\}_{i=0}^{n-1}$, where the points of $S$ are circularly ordered.

There exists $w \in[1, m]_{Z}$ such that

$$
w=\min \left\{t \in[0, m]_{Z} \mid H_{t}(S) \neq S\right\} .
$$

Without loss of generality, $x_{1} \notin H_{w}(S)$. Then the induced function $H_{w-1}$ is a bijection, so there exists $x_{u} \in S$ such that $H\left(x_{u}, w-1\right)=x_{1}$. By Proposition 3.1, $H_{w-1}\left(\left\{x_{(u-1) \bmod n}, x_{(u+1) \bmod n}\right\}\right)=\left\{x_{0}, x_{2}\right\}$, and the continuity property of homotopy implies $H\left(x_{u}, w\right) \in\left\{x_{0}, x_{2}\right\}$. Without loss of generality,

$$
H\left(x_{(u-1) \bmod n}, w-1\right)=x_{0}
$$

and

$$
H\left(x_{u}, w\right)=x_{2} \text {. }
$$

Suppose $n>4$. Equation (2) implies

$H\left(x_{(u-1) \bmod n}, w\right) \in\left\{x_{1}, x_{2}, x_{3}\right\}$, but this is impossible, for the following reasons.

- $H\left(x_{(u-1) \bmod n}, w\right) \neq x_{1}$, by choice of $x_{1}$.

- $H\left(x_{(u-1) \bmod n}, w\right) \notin\left\{x_{2}, x_{3}\right\}$, from Equation (1), because $n>4$ implies neither $x_{2}$ nor $x_{3}$ is $\kappa$-adjacent to $x_{0}$.

The contradiction arose from the assumption that $n>4$. Therefore, we must have $n \leq 4$. Since a digital simple closed curve is assumed to have at least 4 points, we must have $n=4$.

In [8], an example is given of a simple closed $c_{2}$ curve $S \subset Z^{2}$ such that $Z^{2} \backslash S$ has $2 c_{1}$-connected components, with $|S|=4$, such that $S$ is $c_{2}$-contractible (see Figure 2). By contrast, we have the following.

Theorem 3.3 Let $(S, \kappa)$ be a simple closed $\kappa$-curve such that $|S|>4$. Then $S$ is not $\kappa$-contractible.

Proof: It follows from Theorem 3.2 that if $|S|>4$, then there cannot be a $(\kappa, \kappa)$-homotopy between $1_{S}$ and a constant map in $S$.

It is natural to ask whether we can obtain an analog of Theorem 3.3 for higher dimensions. In order to do so, we must decide what is an appropriate digital model for the $k$-dimensional Euclidean sphere $S^{k}$. The literature contains the following.

- Let $X_{2 k}$ be the set of all points $p \in Z^{k}$ such that $p$ is a $c_{1}$-neighbor of the origin.

Then ([10], Proposition 4.1) $X_{2 k}$ is $c_{k}$-contractible. Notice that this example generalizes the contractibility of a 4-point digital simple closed curve [8] (see Figure 2 for the planar version); the contractibility seems due to the smallness of the image, rather than its form.

- Let $B d I_{k}$ denote the boundary of a digital $k$-cube, i.e., for some integer $n>2$,

Bd $I_{k}=\left\{\left(x_{1}, \ldots, x_{k}\right) \in[0, n-1]_{Z}^{k} \mid\right.$ for some $i \in\{1, \ldots, k\}$, $\left.x_{i} \in\{0, n-1\}\right\}$. 


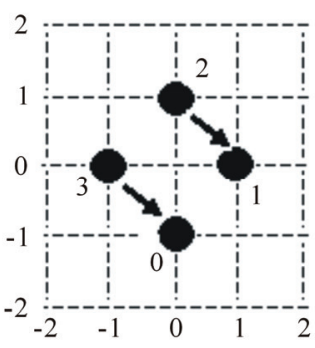

(a)

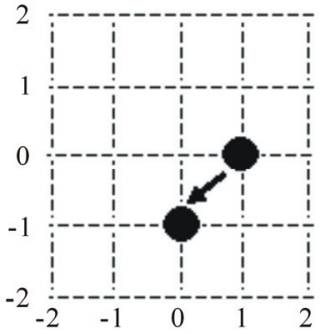

(b)

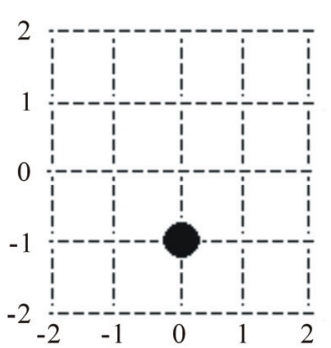

(c)

Figure 2. $c_{2}$-contraction of $X_{4}$, a digital simple closed $c_{2}$-curve, via $H: X_{4} \times[0,2]_{Z} \rightarrow X_{4}$. (a) shows $X_{4}$. Points are labeled by indices. We have $H(x, 0)=x$ for all $x \in X_{4}$. Arrows show the "motion" of $x_{2}, x_{3}$ at the next step. (b) shows the results of the first step of the contraction: $H\left(x_{0}, 1\right)=H\left(x_{3}, 1\right)=x_{0} ; H\left(x_{1}, 1\right)=H\left(x_{2}, 1\right)=x_{1}$. The arrow shows the motion at the next step of the contraction. (c) shows the results of the final step of the contraction: $H\left(x_{i}, 2\right)=x_{0}$ for all $i \in\{0,1,2,3\}$.

See Figure 3 for the planar version. Then ([7], Corollary 5.9) for $n>2, B d I_{k}$ is not $c_{1}$-contractible.

The next result may be interpreted as stating that for $n>4$, a map homotopic to $1_{s}$ must be a "rotation" of the points of $S$.

Theorem 3.4 Let $S$ be a simple closed $\kappa$-curve such that $|S|=n>4$. Let $f: S \rightarrow S$ be a $(\kappa, \kappa)$ continuous function such that $f$ is $(\kappa, \kappa)$-homotopic to $1_{s}$. Then, for some integer $j$, we have

$$
f\left(x_{i}\right)=x_{(i+j) \bmod n} \text { for all } i \in[0, n-1]_{Z} \text {. }
$$

Proof: Let $H: S \times[0, m]_{Z} \rightarrow S$ be a $(\kappa, \kappa)$-homotopy from $1_{S}$ to $f$. For $t \in[0, m]_{Z}$, let $H_{t}: S \rightarrow S$ be the induced map. The assertion follows from the following.

Claim 1: For each $t \in[0, m]_{Z}$, there is an integer $j$ such that $H_{t}\left(x_{i}\right)=x_{(i+j) \bmod n}$ for all $i \in[0, n-1]_{Z}$.

To prove Claim 1, we argue by mathematical induction on $t$. For $t=0$, we can clearly take $j=0$. Now, suppose the claim is valid for $t \in[0, u]_{z}$ such that $0 \leq u<m$. Then, in particular, there is an integer $j$ such that $H_{u}\left(x_{i}\right)=x_{(i+j) \bmod n}$ for all $i \in[0, n-1]_{Z}$. The continuity properties of homotopy imply $H_{u+1}\left(x_{0}\right) \in$ $\left\{x_{(j-1) \bmod n}, x_{j}, x_{(j+1) \bmod n}\right\}$.

Without loss of generality, $H_{u+1}\left(x_{0}\right)=x_{j}$. This is the initial case of the following:

Claim 2: $\quad H_{u+1}\left(x_{k}\right)=x_{(k+j) \bmod n}$ for all $k \in[0, n-1]_{Z}$.

Suppose the equation of Claim 2 is true for all $k \in[0, v]_{Z}$, for some $v \in[0, n-2]_{Z}$. In particular,

$$
H_{u+1}\left(x_{v}\right)=x_{(v+j) \bmod n} .
$$

By the continuity properties of homotopy, $H_{u+1}\left(x_{v+1}\right)$ is adjacent to or coincides with $H_{u+1}\left(x_{v}\right)=x_{(v+j) \bmod n}$ and with $H_{u}\left(x_{v+1}\right)=x_{(v+1+j) \bmod n}$. Thus, $H_{u+1}\left(x_{v+1}\right) \in\left\{x_{(v+j) \bmod n}\right.$, $\left.x_{(v+1+j) \bmod n}\right\}$. Since $H_{u+1}$ must also be an isomorphism by Theorem 3.2, from Equation (3), $H_{u+1}\left(x_{v+1}\right)=$ $x_{(v+1+j) \bmod n}$. This completes the induction proof for the Claim 2, which, in turn, completes the induction proof for the Claim 1. Thus, the assertion is established.

\section{Antipodal Maps}

A classical theorem of Euclidean topology, due to $\mathrm{K}$. Borsuk, states that a continuous antipodal map $f: S^{d}$ $\rightarrow S^{d}$ from the $d$-dimensional unit sphere to itself is not homotopic to a constant map [19]. In this section, we obtain a digital analog, Theorem 4.16, for $d=1$.

We say a set $X \subset Z^{d}$ is symmetric with respect to the origin if $X$ satisfies the property that

$$
x \in X \text { if and only if }-x \in X \text {. }
$$

Suppose we have $X \subset Z^{d_{0}}, Y \subset Z^{d_{1}}$, and $X$ is symmetric with respect to the origin. A function $f: X \rightarrow Y$ is called antipodal-preserving or an

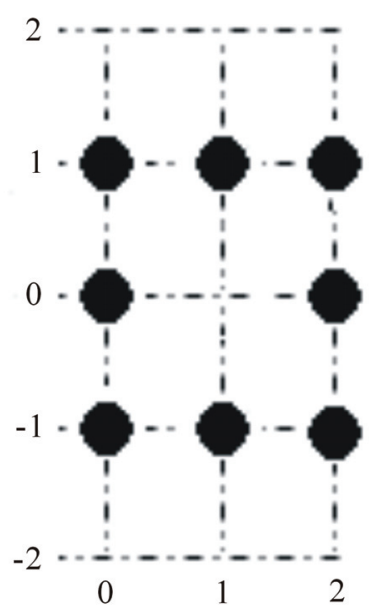

Figure 3. $B d I_{2}$, the "boundary" of a digital square. 


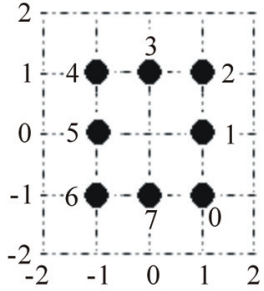

(a)

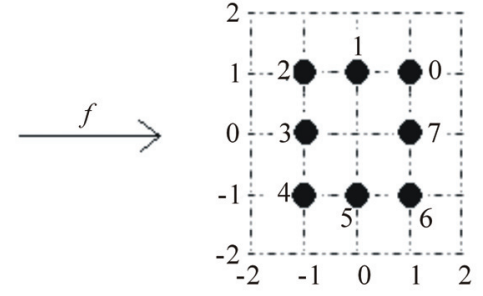

(b)
Figure 4. A digital simple closed $c_{1}$-curve $S=\left\{x_{i}\right\}_{i=0}^{7}$ and a $\left(c_{1}, c_{1}\right)$-continuous map $f: S \rightarrow S$ such that $f$ is $\left(c_{1}, c_{1}\right)$ -homotopic to $1_{S}$. According to Theorem 3.4, such a map $f$ must "rotate" the members of $S$. In (a), points of $S$ are labeled by indices. In (b), each $y \in S$ is labeled by the index of the point $x_{i} \in S$ such that $f\left(x_{i}\right)=y$. Here, we have $f\left(x_{i}\right)=x_{(i+2) \bmod 8}$ for all $i$.

antipodal map if $f(-x)=-f(x)$ for all $x \in X$ [19]. In this section, we study properties of continuous antipodal maps between digital simple closed curves.

Lemma 4.1 Let $p_{0}, p_{1}$ be $c_{l}$-adjacent points in $Z^{d}$, $1 \leq l \leq d$. Then $p_{0}$ and $p_{1}$ are not antipodal.

Proof: The hypothesis implies that there is an index $i$ such that $p_{0}$ and $p_{1}$ differ by 1 in the $i^{\text {th }}$ coordinate: $\left|p_{0, i}-p_{1, i}\right|=1$. If $p_{0}$ and $p_{1}$ are antipodal, this would imply $\left\{p_{0, i}, p_{1, i}\right\}=\{-1 / 2,1 / 2\}$, which is impossible.

Lemma 4.2 Let $p_{0}, p_{1}$ be $c_{l}$-adjacent points in $Z^{d}$, $1 \leq l \leq d$. Then $-p_{0}$ and $-p_{1}$ are $c_{l}$-adjacent.

Proof: Elementary, and left to the reader.

Lemma 4.3 Let $S=\left\{x_{i}\right\}_{i=0}^{n-1}$ be a digital simple closed $c_{l}$-curve in $Z^{d}$ such that the points of $S$ are circularly ordered. If $S$ is symmetric with respect to the origin, then the origin is not a member of $S$.

Proof: Suppose the origin is a member of $S$. Without loss of generality, $x_{0}$ is the origin, and therefore is its own antipode.

By Lemma 4.2, $x_{1}$ and $x_{n-1}$ are antipodes; by Lemma 4.1, these points are not $c_{l}$-adjacent. This establishes the base case of an induction argument: $S_{1}=\left\{x_{i}\right\}_{i=0}^{1} \cup\left\{x_{n-j}\right\}_{j=1}^{1}$ is a connected subset of $S$, such that $x_{1}$ and $x_{n-1}$ are non-adjacent antipodes; hence, $S_{1}$ is $\left(c_{l}, c_{1}\right)$-isomorphic to a digital interval. Now, suppose for some integer $k, \quad 1 \leq k<\lfloor(n-1) / 2\rfloor$, $S_{k}=\left\{x_{i}\right\}_{i=0}^{k} \cup\left\{x_{n-j}\right\}_{j=1}^{k}$ is $\left(c_{l}, c_{1}\right)$-isomorphic to a digital interval, with endpoints $x_{k}$ and $x_{n-k}$, such that $x_{m}$ and $x_{n-m}$ are non-adjacent antipodes for all $m \in\{1, \ldots, k\}$. Then, by Lemma $4.2, x_{k+1}$ and $x_{n-k-1}$ are antipodes, and by Lemma 4.1, these points are not $c_{l}$-adjacent. Thus, $S_{k+1}=\left\{x_{i}\right\}_{i=0}^{k+1} \cup\left\{x_{n-j}\right\}_{j=1}^{k+1}$ is $\left(c_{l}, c_{1}\right)$ isomorphic to a digital interval, with endpoints $x_{1}$ and $x_{n-1}$.
This completes an induction argument from which we conclude that

$$
S^{\prime}=\left\{x_{i}\right\}_{i=0}^{\lfloor(n-1) / 2\rfloor} \cup\left\{x_{i}\right\}_{i=n-\lfloor(n-1) / 2\rfloor}^{n-1}
$$

is $\left(c_{l}, c_{1}\right)$-isomorphic to a digital arc, with the endpoints of $S^{\prime}$ being $x_{\lfloor(n-1) 2\rfloor}$ and $x_{n\lfloor(n-1) / 2\rfloor}$, such that $1 \leq i \leq\lfloor(n-1) / 2\rfloor$ implies $x_{i}$ and $x_{n-i}$ are non-adjacent antipodes.

- If $n$ is odd, then $n-1$ is even, so $S^{\prime}=S$. This is a contradiction, since $S^{\prime}$ is not a simple closed $c_{l}$ curve.

- If $n$ is even, then $S^{\prime}=S \backslash\left\{x_{n 2}\right\}$. Since $S$ is symmetric with respect to the origin, we must have that $x_{n / 2}$ is the origin. But since $S$ is a simple closed $c_{l}$-curve in which $x_{0}$ is the origin, this is a contradiction.

Whether $n$ is even or odd, the assumption that the origin belongs to $S$ yields a contradiction. Hence, the origin is not a member of $S$.

Lemma 4.4 Let $S=\left\{x_{i}\right\}_{i=0}^{n-1}$ be a digital simple closed $c_{l}$-curve in $Z^{d}$ such that the points of $S$ are circularly ordered. If $S$ is symmetric with respect to the origin, then $n$ is even.

Proof: By Lemma 4.3, the origin is not a member of $S$, so every member of $S$ is distinct from its antipode. Therefore, $n$ must be even.

Lemma 4.5 Let $S=\left\{x_{i}\right\}_{i=0}^{n-1}$ be a digital simple closed $c_{l}$-curve in $Z^{d}$ such that the points of $S$ are circularly ordered. If $S$ is symmetric with respect to the origin, then for all $i$ we have $-x_{i}=x_{(i+n / 2) \bmod n}$.

Proof: Suppose there is a simple closed $c_{l}$-curve $S=\left\{x_{i}\right\}_{i=0}^{n-1}$ that is symmetric with respect to the origin such that the points of $S$ are circularly ordered, such that there exist indices $u, v$ such that $x_{u}$ and $x_{v}$ are antipodes and $v \neq(u+n / 2) \bmod n$. Without loss of generality, we can assume $u=0, v \neq n / 2$.

Then, from Lemma 4.2, $x_{1}$ is antipodal to either $x_{v-1}$ or $x_{(v+1) \bmod n}$. Without loss of generality, $x_{1}$ and $x_{v-1}$ are antipodal. If $v-1=1$, this is a contradiction of Lemma 4.3; or, if $v-1=2$, this is a contradiction of Lemma 4.1; otherwise, we inductively repeat the argument above with the antipodal (by Lemma 4.2) pair $\left(x_{2}, x_{v-2}\right)$, etc., until similarly we obtain a contradiction of Lemma 4.3 or of Lemma 4.1. The assertion follows.

We have the following.

Theorem 4.6 Let $S_{i} \subset Z^{d_{i}}$ be simple closed $\kappa_{i}$-curves, $i \in\{0,1\}$, each symmetric with respect to the origin. Let $f: S_{0} \rightarrow S_{1}$ be a $\left(\kappa_{0}, \kappa_{1}\right)$-continuous antipodal map. Then $f$ is onto.

Proof: Let $S_{1}=\left\{x_{i}\right\}_{i=0}^{n-1}$, where the points of $S_{1}$ are circularly ordered. Without loss of generality, there exists $p \in S_{0}$ such that $f(p)=x_{0}$. Since $f$ is anti- 
podal, it follows from Lemma 4.5 that $f(-p)=x_{n / 2}$. Since $f$ is continuous and $S_{0}$ is $\kappa_{0}$-connected, it follows that one of the $\kappa_{1}$-paths in $S_{1}$ from $x_{0}$ to $x_{n / 2}$ is contained in $f\left(S_{0}\right)$. Without loss of generality, $\left\{x_{j}\right\}_{j=0}^{n / 2} \subset f\left(S_{0}\right)$. For $n / 2<j<n$, there exists $q \in S_{0}$ such that $f(q)=x_{j-n / 2}$, and from Lemma 4.5,

$x_{j}=-x_{j-n / 2}=-f(q)$

$=($ since $f$ is antipodal $) f(-q) \in f\left(S_{0}\right)$.

Thus, $f\left(S_{0}\right)=S_{1}$.

Corollary 4.7 Let $S_{i} \subset Z^{d_{i}}$ be simple closed $\kappa_{i}$ curves, $i \in\{0,1\}$, each symmetric with respect to the origin. Let $f: S_{0} \rightarrow S_{1}$ be a $\left(\kappa_{0}, \kappa_{1}\right)$-continuous antipodal map. If $\left|S_{0}\right|=\left|S_{1}\right|$, then $f$ is a $(\kappa, \kappa)$-isomorphism.

Proof: By Theorem 4.6, $f$ is onto. The assertion follows from Proposition 3.1.

Proposition 4.8 Let $X=\left\{x_{i}\right\}_{i=0}^{n_{X}-1}$ be a simple closed $\kappa_{X}$-curve with circularly ordered points. Let $Y=$ $\left\{y_{i}\right\}_{i=0}^{n_{Y}-1}$ be a simple closed $\kappa_{Y}$-curve with circularly ordered points. Suppose $H: X \times[0, m]_{Z} \rightarrow Y$ is a $\left(\kappa_{X}, \kappa_{Y}\right)$-homotopy between the $\left(\kappa_{X}, \kappa_{Y}\right)$-continuous maps $H_{0}, H_{m}$ such that $H_{0}\left(x_{0}\right)=H_{m}\left(x_{0}\right)$. Then there is a $\left(\kappa_{X}, \kappa_{Y}\right)$-homotopy $G: X \times[0, m]_{Z} \rightarrow Y$ between $H_{0}$ and $H_{m}$ such that $G\left(x_{0}, t\right)=H_{0}\left(x_{0}\right)$ for all $t \in[0, m]_{Z}$

Proof: Without loss of generality, $H_{0}\left(x_{0}\right)=y_{0}$. Let $a:[0, m]_{Z} \rightarrow[0, m]_{Z}$ be defined by $a(t)=i$ if $H\left(x_{0}, t\right)=y_{i}$.

Let $G: X \times[0, m]_{Z} \rightarrow Y$ be defined by

$$
G\left(x_{i}, t\right)=y_{[j-a(t)] \bmod n Y}, \text { if } H\left(x_{i}, t\right)=y_{j} .
$$

Roughly, we may think of $G\left(x_{i}, t\right)$ as rotating $H\left(x_{i}, t\right)$ counter to the rotation of $H\left(x_{0}, t\right)$, so that the image under $G$ of $x_{0}$ at time $t$ is constant with respect to $t$. We show below that $G$ is a homotopy. In particular, $\quad a(0)=a(m)=0 \quad, \quad$ so $\quad G_{0}=H_{0} \quad$ and $G_{m}=H_{m} ;$ and, for all $t \in[0, m]_{Z}$,

$$
G\left(x_{0}, t\right)=y_{[a(t)-a(t)] \bmod n_{Y}}=y_{0} .
$$

For each $x_{i} \in X$ and $t \in[0, m]_{Z}$, if $H_{t}\left(x_{i}\right)=y_{j}$ then we have the following.

- By the continuity of $H_{t}$, we have

$$
H_{t}\left(\left\{x_{(i-1) \bmod n_{X}}, x_{(i+1) \bmod n_{X}}\right\}\right) \subset\left\{y_{(j-1) \bmod n_{Y}}, y_{j}, y_{(j+1) \bmod n_{Y}}\right\} \text {. }
$$

Therefore, $G_{t}\left(x_{i}\right)=y_{[j-a(t)] \bmod n_{Y}}$ and

$$
\begin{aligned}
& G_{t}\left(\left\{x_{(i-1) \bmod n_{X}}, x_{(i+1) \bmod n_{X}}\right\}\right) \subset \\
& \left\{y_{[j-1-a(t)] \bmod n_{Y}}, y_{[j-a(t)] \bmod n_{Y}}, y_{[j+1-a(t)] \bmod n_{Y}}\right\} .
\end{aligned}
$$

Therefore, $G_{t}$ is $\left(\kappa_{X}, \kappa_{Y}\right)$-continuous.

- Let $G_{x_{i}}:[0, m]_{Z} \rightarrow Y$ be the induced function defined by $G_{x_{i}}(t)=G\left(x_{i}, t\right)$. To simplify the following, let
$G_{x_{i}}(-1)=H_{-1}\left(x_{i}\right)=H_{0}\left(x_{i}\right)$,

$G_{x_{i}}(m+1)=H_{m+1}\left(x_{i}\right)=H_{m}\left(x_{i}\right)$,

$a(-1)=a(0)=0=a(m)=a(m+1)$. Note that the continuity of $H_{x_{0}}$ implies that

$$
\{a(t-1), a(t+1)\} \subset\{a(t)-1, a(t), a(t)+1\} .
$$

Suppose $H_{t}\left(x_{i}\right)=y_{j}$, so $G_{x_{i}}(t)=y_{[j-a(t)] \bmod n_{Y}}$. If $a(t-1)=a(t)-1$, then

$G_{x_{i}}(t-1)=y_{[j-a(t-1)] \bmod n_{Y}}=y_{[j-a(t)+1] \bmod n_{Y}}$ is $\kappa_{Y}$-adjacent to $G_{x_{i}}(t)$.

If $a(t-1)=a(t)$, then $G_{x_{i}}(t-1)=G_{x_{i}}(t)$.

If $a(t-1)=a(t)+1$, then

$G_{x_{i}}(t-1)=y_{[j-a(t-1)] \bmod n_{Y}}=y_{[j-a(t)-1] \bmod n_{Y}}$ is $\kappa_{Y}$-adjacent to $G_{x_{i}}(t)$.

Similarly, $G_{x_{i}}(t+1)$ is $\kappa_{Y}$-adjacent to, or equal to, $G_{x_{i}}(t)$. Therefore, the induced function $G_{x_{i}}$ is $\left(c_{1}, \kappa_{Y}\right)$ continuous.

Therefore, $G$ is a homotopy between $H_{0}$ and $H_{m}$ such that $G\left(x_{0}, t\right)=H_{0}\left(x_{0}\right)$ for all $t \in[0, m]_{Z}$.

Let $\left(X, \kappa_{X}\right)$ and $\left(Y, \kappa_{Y}\right)$ be digital images and let $y \in Y$. We denote by $\bar{y}: X \rightarrow Y$ (or, for short, $\bar{y}$ ) the constant map $\bar{y}(x)=y$ for all $x \in X$.

Lemma 4.9 Let $\left(X, \kappa_{X}\right)$ and $\left(Y, \kappa_{Y}\right)$ be digital simple closed curves and let $f: X \rightarrow Y$ be $\left(\kappa_{X}, \kappa_{Y}\right)$ homotopic to a constant map. Then for any $x_{0} \in X$, $f:\left(X, x_{0}\right) \rightarrow\left(Y, f\left(x_{0}\right)\right)$ is $\left(\kappa_{X}, \kappa_{Y}\right)$-pointed homotopic to the constant map $\overline{f\left(x_{0}\right)}$.

Proof: Let $F: X \times\left[0, m_{0}\right]_{Z} \rightarrow Y$ be a $\left(\kappa_{X}, \kappa_{Y}\right)-$ homotopy between $f$ and the constant map $\bar{y}_{0}$ for some $y_{0} \in Y$. Since $Y$ is $\kappa_{Y}$-connected, there is a path $p:\left[0, m_{1}\right]_{Z} \rightarrow Y$ from $y_{0}$ to $f\left(x_{0}\right)$. Then the map $H: X \times\left[0, m_{0}+m_{1}\right]_{Z} \rightarrow Y$ defined by

$$
H(x, t)= \begin{cases}F(x, t) & \text { if } 0 \leq t \leq m_{0} ; \\ p\left(t-m_{0}\right) & \text { if } m_{0} \leq t \leq m_{0}+m_{1},\end{cases}
$$

is a homotopy between $f$ and $\overline{f\left(x_{0}\right)}$. The assertion follows from Proposition 4.8.

Given a digital image $(X, \kappa)$ and a positive integer $n$, for $x \in X$ we define [20]

$$
\begin{aligned}
& N_{\kappa}(x, n)=\{x\} \cup \\
& \{y \in X \mid \text { there is a } \kappa \text {-path in } X \\
& \text { from } y \text { to } x \text { of length at most } n\} .
\end{aligned}
$$

The covering space and the lifting of maps are notions borrowed from algebraic topology that have been important in digital algebraic topology. We have the following.

Definition 4.10 [20] Let $\left(E, \kappa_{E}\right)$ and $\left(B, \kappa_{B}\right)$ be digital images. Let $p: E \rightarrow B$ be a $\left(\kappa_{E}, \kappa_{B}\right)$-continuous function. Suppose for each $b \in B$ there exists $\varepsilon \in N$ such that 
- for some $\delta \in N$ and some index set $M$, $p^{-1}\left(N_{\kappa_{B}}(b, \varepsilon)\right)=\bigcup_{i \in M} N_{\kappa_{E}}\left(e_{i}, \delta\right)$ with $e_{i} \in p^{-1}(b) ;$

- if $i, j \in M, i \neq j$, then

$N_{\kappa_{E}}\left(e_{i}, \delta\right) \cap N_{\kappa_{E}}\left(e_{j}, \delta\right)=\varnothing$; and

- the restriction map $\left.p\right|_{N_{\kappa_{E}}\left(e_{i}, \delta\right)}: N_{\kappa_{E}}\left(e_{i}, \delta\right) \rightarrow N_{\kappa_{B}}(b, \varepsilon)$ is a $\left(\kappa_{E}, \kappa_{B}\right)$-isomorphism for all $i \in M$.

Then the map $p$ is a $\left(\kappa_{E}, \kappa_{B}\right)$ - covering map, and the pair $(E, p)$ is a $\left(\kappa_{E}, \kappa_{B}\right)$ - covering (or covering space).

The following is a somewhat simpler characterization of the digital covering than given in Definition 4.10.

Theorem 4.11 [14] Let $\left(E, \kappa_{E}\right)$ and $\left(B, \kappa_{B}\right)$ be digital images. Let $p: E \rightarrow B$ be a $\left(\kappa_{E}, \kappa_{B}\right)-$ continuous function. Then the map $p$ is a $\left(\kappa_{E}, \kappa_{B}\right)-$ covering map if and only if for each $b \in B$, there is an index set $M$ such that

- $p^{-1}\left(N_{\kappa_{B}}(b, 1)\right)=\cup_{i \in M} N_{\kappa_{E}}\left(e_{i}, 1\right)$, with $e_{i} \in p^{-1}(b)$;

- if $i, j \in M, i \neq j$, then $N_{\kappa_{E}}\left(e_{i}, 1\right) \cap N_{\kappa_{E}}\left(e_{j}, 1\right)=\varnothing$; and

- the restriction map $\left.p\right|_{N_{\kappa_{E}}\left(e_{i}, 1\right)}: N_{\kappa_{E}}\left(e_{i}, 1\right) \rightarrow N_{\kappa_{B}}(b, 1)$ is a $\left(\kappa_{E}, \kappa_{B}\right)$-isomorphism for all $i \in M$.

The following is a minor generalization of an example of a digital covering map given in [20].

Example 4.12 Let $C=\left\{c_{i}\right\}_{i=0}^{m-1} \subset Z^{d}$ be a circularly ordered simple closed $\kappa$-curve. Let $p: Z \rightarrow C$ be defined by $p(z)=z \bmod m$ for all $z \in Z$. Then $p$ is $a\left(c_{1}, \kappa\right)$-covering map (see Figure 5).

Definition 4.13 [20] For digital images $\left(E, \kappa_{E}\right)$, $\left(B, \kappa_{B}\right)$, and $\left(X, \kappa_{X}\right)$, let $p: E \rightarrow B$ be a $\left(\kappa_{E}, \kappa_{B}\right)$ covering map, and let $f: X \rightarrow B$ be $\left(\kappa_{X}, \kappa_{B}\right)$ continuous. $A$ lifting of $f$ with respect to $p$ is a $\left(\kappa_{X}, \kappa_{E}\right)$-continuous function $F: X \rightarrow E$ such that $p \circ F=f$.

See Figure 6 for an illustration of Definition 4.13.

Theorem 4.14 [20] Let $\left(E, \kappa_{E}\right)$ be a digital image and $e_{0} \in E$. Let $\left(B, \kappa_{B}\right)$ be a digital image and $b_{0} \in B$. Let $p: E \rightarrow B$ be a $\left(\kappa_{E}, \kappa_{B}\right)$-covering map such that $p\left(e_{0}\right)=b_{0}$. Then a $\kappa_{B}$-path $f:[0, m]_{Z} \rightarrow B$ beginning at $b_{0}$ has a unique lifting with respect to $p$ to a path $\widetilde{f}$ in $E$ starting at $e_{0}$.

For a positive integer $n$, a $\left(\kappa_{E}, \kappa_{B}\right)$-covering map $p: E \rightarrow B$ is called a radius $n$ local isomorphism [21] if for each $b \in B$ and $e \in p^{-1}(b),\left.p\right|_{N_{\kappa_{E}}(e, n)}$ $\rightarrow N_{\kappa_{B}}(b, n)$ is an isomorphism. It was observed in [14] that every covering is a radius 1 local isomorphism, but there are coverings that are not radius 2 local isomorphisms.

Theorem 4.15 [21] Let $\left(E, \kappa_{E}\right)$ be a digital image and $e_{0} \in E$. Let $\left(B, \kappa_{B}\right)$ be a digital image and $b_{0} \in B$. Let $p: E \rightarrow B$ be a $\left(\kappa_{E}, \kappa_{B}\right)$-covering map such that $p\left(e_{0}\right)=b_{0}$. Suppose $p$ is a radius 2 local isomorphism. For $\kappa_{E}$-paths $g_{0}, g_{1}:[0, m]_{Z} \rightarrow E$ that start at $e_{0}$, if there is a $\kappa_{B}$-homotopy in $B$ from $p \circ g_{0}$ to $p \circ g_{1}$ that holds the endpoints fixed, then $g_{0}(m)=g_{1}(m)$, and there is a $\kappa_{E}$-homotopy in $E$ from $g_{0}$ to $g_{1}$ that holds the endpoints fixed.

Theorem 4.16 Let $X=\left\{x_{i}\right\}_{i=0}^{n_{X}-1}$ be a simple closed $\kappa_{X}$-curve with points circularly ordered, that is symmetric with respect to the origin. Let $Y=\left\{y_{i}\right\}_{i=0}^{n_{Y}-1}$ be a simple closed $\kappa_{Y}$-curve with points circularly ordered, that is symmetric with respect to the origin. Let $f: X \rightarrow Y$ be a $\left(\kappa_{X}, \kappa_{Y}\right)$-continuous antipodal map. If $|Y|>5$, then $f$ is not $\left(\kappa_{0}, \kappa_{1}\right)$-nullhomotopic.

Proof: Suppose there is such a function $f$ that is $\left(\kappa_{0}, \kappa_{1}\right)$-nullhomotopic. From Lemma 4.9, $f$ is point- ed homotopic to $\overline{f\left(x_{0}\right)}$.

Let $b:\left[0, n_{X}\right]_{Z} \rightarrow X$ be defined by $b(t)=x_{t \bmod n_{X}}$. Let $p: Z \rightarrow Y$ be the $\left(c_{1}, \kappa_{Y}\right)$-covering map defined by $p(z)=y_{z \bmod n_{Y}}$ (see Example 4.12). By Proposition 2.2, $f \circ b$ and $\overline{f\left(x_{0}\right)} \circ b$ are $\left(c_{1}, \kappa_{Y}\right)$-homotopic paths in $Y$. From Theorem 4.14, these functions have unique

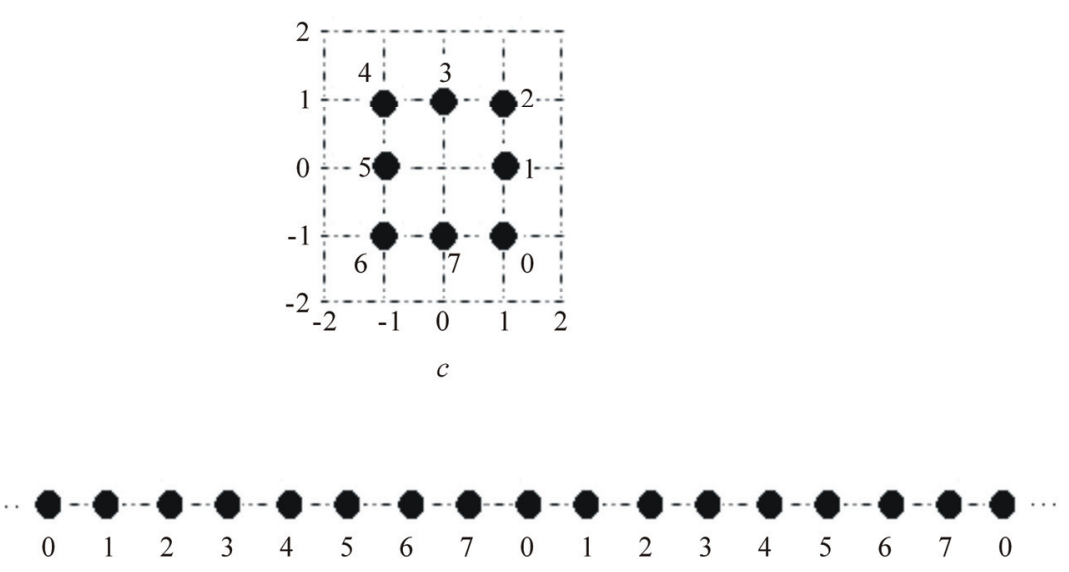

Figure 5. A simple closed $c_{1}$-curve $C$ and a covering by the digital line $Z$. Members of $C$ are labeled by their respective indices. A point $z \in Z$ is labeled by the index of point of $C$ to which the covering map sends $z$. 

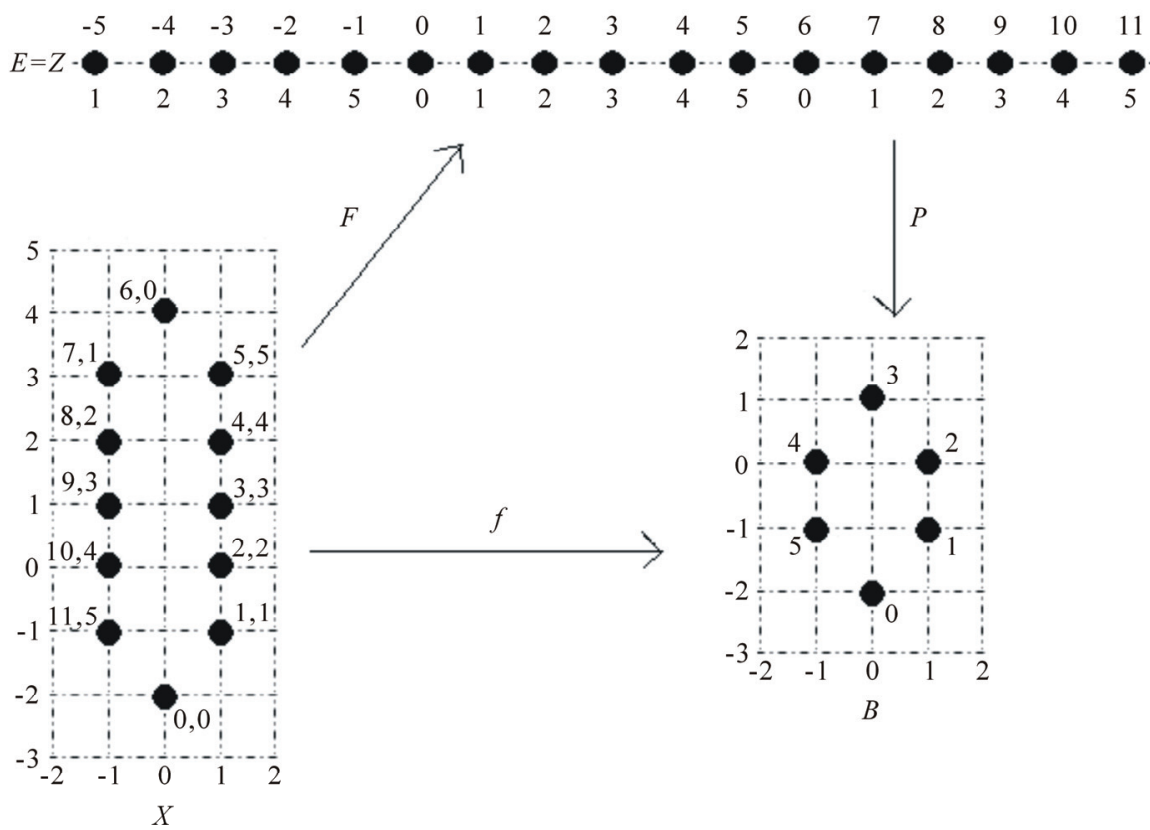

Figure 6. Example of lifting. $B=\left\{b_{i}\right\}_{i=0}^{5}$ is a simple closed $c_{2}$-curve whose members are labeled by their indices. $E=Z$ has its points $z$ labeled above by their coordinates and labeled below by the index $i$ such that $p(z)=b_{i}$ (note $p$ is given by the formula $\left.p(z)=b_{z \bmod 6}\right) . \quad X=\left\{x_{m}\right\}_{m=0}^{11}$ is a simple closed $c_{2}$-curve that has points labeled by a pair $m, n$ such that $m$ is the index of the point, $f\left(x_{m}\right)=b_{n}$ (thus, $f$ is defined by $\left.f\left(x_{m}\right)=b_{m \bmod 6}\right)$, and $F\left(x_{m}\right)=m$. Since $p$ is a covering map (by Example 4.12) and $p \circ F=f, F$ is a lifting of $f$ with respect to $p$.

liftings with respect to $p$ to paths $F_{0}$ and $F_{1}$, respectively, in $Z$, each starting at $0 \in p^{-1}(f(b(0)))$. Since $|Y|>5, N_{\kappa Y}\left(f\left(x_{0}\right), 2\right) \neq Y$, so $p$ is a radius 2 local isomorphism. From Theorem 4.15, $F_{0}$ and $F_{1}$ must end at the same point. Indeed, this point must be 0 , for the uniqueness of $F_{1}$ implies $F_{1}$ must be the constant map $\overline{0}$.

Since $f$ is an antipodal map, we must have $\left|F_{0}\left(t+n_{X} / 2\right)-F_{0}(t)\right|=n_{Y} / 2$ for all $t \in\left[0, n_{X} / 2-1\right]_{Z}$.

Since $F_{0}(0)=0, F_{0}\left(n_{X} / 2\right) \in\left\{-n_{Y} / 2, n_{Y} / 2\right\}$. Without loss of generality,

$$
F_{0}\left(n_{X} / 2\right)=n_{Y} / 2
$$

Since $F$ is continuous and $n_{Y}>5$, a simple induction argument based on Equations (4) and (5) shows that $F_{0}\left(t+n_{X} / 2\right)>F_{0}(t)$ for all $t \in\left[0, n_{X} / 2-1\right]_{Z}$. But since $F_{0}\left(n_{X}\right)=0$, this implies with Equation (4) that $F_{0}\left(n_{X} / 2\right)=-n_{Y} / 2$, which contradicts Equation (5). The assertion follows from the contradiction.

\section{Antipodes Mapped Together}

A classical result of topology is that if $f$ is a continuous map from the $d$-dimensional unit sphere $S^{d}$ to Euclidean $d$-space $R^{d}$, then there is a pair of antipodes $x,-x \in S^{d}$ such that $f(x)=f(-x)$ [17]. For $d=1$, the following is a digital analog.

Theorem 5.1 Let $S$ be a digital simple closed $c_{l}$-curve with $S=\left\{x_{i}\right\}_{i=0}^{n-1}$, where the points of $S$ are circularly ordered. Suppose $S$ is symmetric with respect to the origin. Suppose $f: S \rightarrow Z$ is a $\left(c_{l}, c_{1}\right)$ continuous function. Then there is a pair of antipodes $x,-x \in S$ such that $|f(x)-f(-x)| \leq 1$.

Proof: By Lemma 4.4, $n$ is even. Consider the function $g:[0, n-1]_{Z} \rightarrow Z$ defined by $g(i)=f\left(x_{i}\right)-$ $f\left(x_{(i+n / 2) \bmod n}\right)$. By Lemma 4.5, we are done if, for some $i, g(i)=0$. Therefore, we assume for all $i$ that

$$
g(i) \neq 0 \text {. }
$$

Clearly, for all $i$,

$$
g[(i+n / 2) \bmod n]=-g(i) .
$$

The continuity of $f$ implies that

$$
|g(i)-g(i+1)| \leq 2 \text {. }
$$

It follows from Equation (7) that $g$ takes both positive and negative values, so from inequality (6), there is an index $j$ such that $g(j)$ and $g(j+1)$ have opposite sign; without loss of generality, $g(j)>0$ and $g(j+1)<0$. From inequality (8), it follows that 


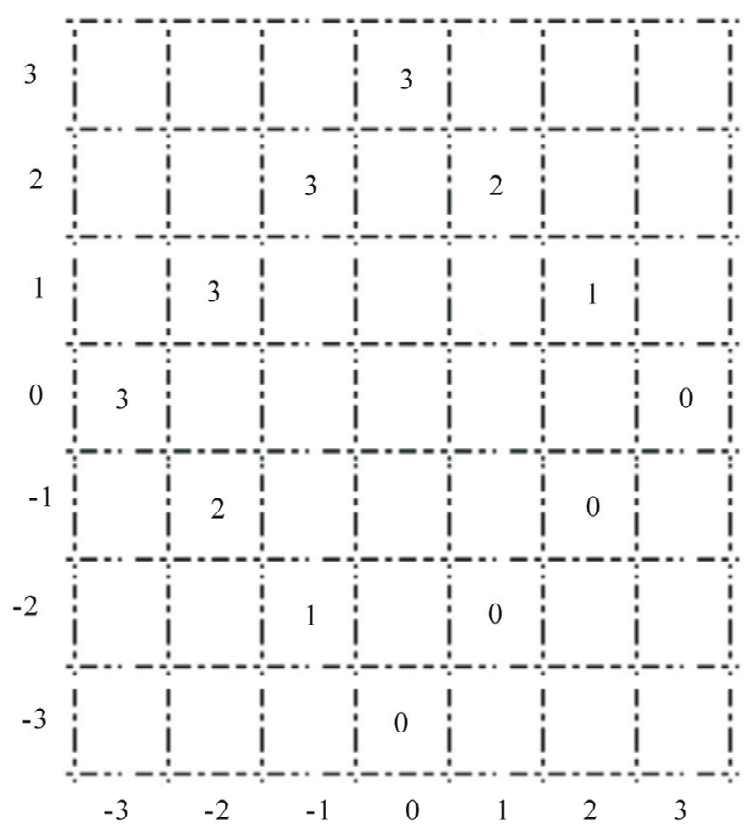

Figure 7. $S$ and $f: S \rightarrow Z$. Each number in the grid labels a point of $S$, showing the image of the grid point under $f$. Note for $s_{0}=(1,2),\left|f\left(s_{0}\right)-f\left(-s_{0}\right)\right|=1$, but there is no $s \in S$ for which $f(s)=f(-s)$.

$g(j)=1$, and the assertion follows.

Theorem 5.1 parallels, in a sense, a result of [2]: In the Euclidean line, a continuous function mapping an interval to itself has a fixed point; in the digital world, a $\left(c_{1}, c_{1}\right)$ continuous function $f:[a, b]_{Z} \rightarrow[a, b]_{Z}$ has a "nearfixed" point, i.e., a point $x$ such that $|x-f(x)| \leq 1$.

That we cannot, in general, conclude the existence of antipodes mapped to the same point in Theorem 5.1, is illustrated in the following example (note the simple closed curve has more than 4 points). Let $S=$ $\{(x, y)|| x|+| y \mid=3\}$. Then $S$ is a simple closed $c_{2}$ curve in $Z^{2}$. The function $f: S \rightarrow Z$ given by

$$
\begin{aligned}
& f(-3,0)=f(-2,1)=f(-1,2)=f(0,3)=3, \\
& f(-2,-1)=f(1,2)=2, \\
& f(-1,-2)=f(2,1)=1, \\
& f(0,-3)=f(1,-2)=f(2,-1)=f(3,0)=0,
\end{aligned}
$$

is a $\left(c_{2}, c_{1}\right)$-continuous function such that $|f(x)-f(-x)|$ $\neq 0$ for each $x \in S$. See Figure 7.

\section{Further Remarks}

In this paper, we have obtained several analogs of classical theorems of Euclidean topology concerning maps on digital simple closed curves. We have shown that digital simple closed curves of more than 4 points are not contractible; that a continuous antipodal map from a digital simple closed curve to itself is not nullhomotopic; and that a continuous map from a digital simple closed curve to the digital line must map a pair of antipodes within 1 of each other. Except as indicated concerning whether or not a digital model of a sphere is contractible, it is not known at the current writing whether these results extend to higher dimensional digital models of Euclidean spheres.

We thank the anonymous referees for their helpful suggestions.

\section{References}

[1] A. Rosenfeld, "Digital Topology," American Mathematical Monthly, Vol. 86, 1979, pp. 76-87.

[2] A. Rosenfeld, "Continuous Functions on Digital Pictures,” Pattern Recognition Letters, Vol. 4, No. 3, 1986, pp. 177-184.

[3] A. Rosenfeld, "Directions in Digital Topology," 11th Summer Conference on General Topology and Applications, 1995. http://atlas-conferences.com/cgi-bin/abstract/ caaf-71

[4] Q. F. Stout, "Topological Matching," Proceedings 15th Annual Symposium on Theory of Computing, Boston, 1983, pp. 24-31.

[5] T. Y. Kong, “A Digital Fundamental Group,” Computers and Graphics, Vol. 13, No. 1, 1989, pp. 159-166.

[6] T. Y. Kong, A. W. Roscoe and A. Rosenfeld, "Concepts of Digital Topology," Topology and Its Applications, Vol. 46, No. 3, 1992, pp. 219-262.

[7] L. Boxer, "Digitally Continuous Functions," Pattern Recognition Letters, Vol. 15, No. 8, 1994, pp. 833-839.

[8] L. Boxer, "A Classical Construction for the Digital Fundamental Group," Journal of Mathematical Imaging and Vision, Vol. 10, No. 1, 1999, pp. 51-62.

[9] T. Y. Kong and A. Rosenfeld, "Topological Algorithms for Digital Image Processing," Elsevier, New York, 1996.

[10] L. Boxer, "Homotopy Properties of Sphere-Like Digital Images," Journal of Mathematical Imaging and Vision, Vol. 24, No. 2, 2006, pp. 167-175.

[11] G. T. Herman, "Oriented Surfaces in Digital Spaces," CVGIP: Graphical Models and Image Processing, Vol. 55, No. 1, 1993, pp. 381-396.

[12] L. Chen, "Gradually Varied Surfaces and Its Optimal Uniform Approximation," SPIE Proceedings, Bellingham, Vol. 2182 1994, pp. 300-307.

[13] L. Chen, "Discrete Surfaces and Manifolds," Scientific Practical Computing, Rockville, 2004.

[14] L. Boxer, "Digital Products, Wedges, and Covering Spaces," Journal of Mathematical Imaging and Vision, Vol. 25, 2006, pp. 159-171.

[15] U. Eckhardt and L. Latecki, "Digital Topology," In: 
Current Topics in Pattern Recognition Research, Research Trends, Council of Scientific Information, 1994. http://cosmic.rrz.uni-hamburg.de/webcat/mathematik/eck hardt/eck00001/eck00001.pdf

[16] R. O. Duda, P. E. Hart and J. H. Munson, "Graphical Data Processing Research Study and Experimental Investigation," Descriptive Note: Quarterly Report No. 7, March 1967, pp. 28-30.

[17] E. Khalimsky, "Motion, Deformation, and Homotopy in Finite Spaces," Proceedings IEEE International Conference on Systems, Man, and Cybernetics, Boston, 1987, pp. 227-234.
[18] L. Boxer, "Properties of Digital Homotopy," Journal of Mathematical Imaging and Vision, Vol. 22, No. 1, 2005, pp. 19-26.

[19] J. Dugundji, “Topology,” Allyn and Bacon, Inc., Boston, 1966.

[20] S. E. Han, "Non-Product Property of the Digital Fundamental Group," Information Sciences, Vol. 171, No. 1-3, 2005, pp. 73-91.

[21] S. E. Han, "Digital Coverings and Their Applications," Journal of Applied Mathematics and Computing, Vol. 18, No. 1-2, 2005, pp. 487-495. 